\title{
Questionário de Permanência Acadêmica: Adaptação Cultural e Evidências de Validade
}

\author{
Jaisso Vautero ${ }^{1}$ (D) Luciane Pozobon (D) \\ Universidade Federal de Santa Maria, Santa Maria-RS, Brasil \\ Ana Daniela Silva (1D \\ Universidade do Minho, Braga, Portugal
}

\section{RESUMO}

O presente estudo focou a questão da permanência universidade, e apresenta dois objetivos principais, o primeiro, de ordem psicométrica, é voltado para adaptação e busca de evidências de validade de um instrumento destinado a avaliação da permanência nas universidades, o Questionário de Permanência Acadêmica (QPA). O segundo objetivo apresenta uma aplicação do instrumento ao verificar se os serviços destinados a aumentar as condições de permanência na universidade estudada poderiam ter algum efeito sobre os escores obtidos no instrumento. Análises fatoriais confirmatórias e testes de confiabilidade indicam boas características psicométricas do instrumento. A estrutura de seis dimensões proposta no estudo original foi confirmada. Quanto aos serviços de apoio à permanência oferecido aos estudantes, os resultados indicam que a percepção de obstáculos no acesso a esses serviços e a utilização do apoio psicossocial e pedagógico foram associadas a baixos resultados no QPA. Tendo em vista que as universidades podem diferir em relação aos serviços com foco na permanência que ofertam, indica-se a aplicação do QPA em diferentes universidades.

Palavras-chave: estudantes universitários; evasão; validade fatorial; assistência estudantil

\section{ABSTRACT - College persistence questionnaire: cultural adaptation and evidence of validity}

The present study focused on academic persistence and had two main aims, the first had a psychometric nature and aimed to adapt of an instrument designed to evaluate academic persistence, the College Persistence Questionnaire (CPQ), and seek evidence of its validity. The second aim was to present a practical use of the instrument, aiming to verify whether the services offered to increase the conditions of persistence in college could have any effect on the students' scores in the instrument. Confirmatory factor analyses and reliability tests indicated good psychometric properties of the instrument. The six-dimensional structure proposed in the original study was confirmed. Regarding the persistence support services offered to the students, the results indicate that the perception of obstacles in the access to these services and the presence of psychosocial and pedagogical support were associated with low results in the CPQ. Given that universities may differ in relation to the persistence-focused services they offer, the investigation using the CPQ in different universities is indicated.

Keywords: college students; persistence; factorial validity; student support services.

\section{RESUMEN - Cuestionario de permanencia académica: adaptación cultural y evidencias de validez}

El presente estudio se centró en el tema de la permanencia universitaria y presenta dos objetivos principales. El primero es de carácter psicométrico y está orientado a adaptar y buscar evidencias de validez del Cuestionario de Permanencia Académica (QPA), un instrumento dirigido a evaluar la permanencia universitaria. El segundo objetivo presenta una aplicación del instrumento para verificar si los servicios destinados a aumentar las condiciones de permanencia en la universidad estudiada podrían tener algún efecto en las puntuaciones obtenidas en el instrumento. Los análisis factoriales confirmatorios y los tests de confiabilidad indicaron buenas características psicométricas del instrumento. Se confirmó la estructura de seis dimensiones propuesta en el estudio original. En cuanto a los servicios de apoyo a la permanencia ofrecidos a los estudiantes, los resultados indican que la percepción de obstáculos en el acceso a estos servicios y el uso del apoyo psicosocial y pedagógico se asociaron con bajos resultados en el QPA. Teniendo en cuenta que las universidades pueden diferir en relación a los servicios centrados en la permanencia ofertados, es recomendable la utilización del QPA en diferentes universidades.

Palabras clave: estudiantes universitarios; permanencia; validez factorial; asistencia estudiantil.

As transformações ocorridas na sociedade brasileira, sobretudo ao longo desta década, fizeram com que a educação superior fosse demandada por um número cada vez maior de pessoas. $\mathrm{O}$ atual panorama do ensino superior no país mostra que se encontra em expansão, ainda que em ritmo menor nos últimos anos (INEP, 2016). Com 
isso, observa-se o aumento das preocupações em torno da permanência do estudante na universidade (Costa, Bispo, \& Pereira, 2018) bem como a necessidade de estudos e instrumentos a fim de avaliar esse comportamento (Santos Júnior \& Real, 2017). O presente estudo foca a questão da permanência, cujo maior obstáculo é a evasão da universidade, garantir a permanência é também combater a evasão (Matta, Lebrão, \& Heleno, 2017). Nesse sentido, seus objetivos principais são dois, o primeiro, de ordem psicométrica, é voltado para adaptação e busca de evidência de validade de um instrumento destinado a avaliação da permanência nas universidades. $\mathrm{O}$ segundo objetivo já traz uma aplicação do instrumento ao procurar uma associação entre este e os serviços de assistência estudantil, destinados a aumentar as condições de permanência nas universidades.

Conceitualmente, a evasão refere-se a uma postura ativa do aluno que decide desligar-se da universidade por sua própria responsabilidade (Matta et al., 2017). Nesse sentido, algumas revisões sobre o tema (e.g., Matta et.al, 2017; Santos Júnior \& Real, 2017) apontam para três fatores como motivadores da evasão: relacionados aos estudantes; à instituição e ao curso, questões socioculturais e econômicos externas (Santos Júnior \& Real, 2017). A definição de permanência pode ser mais complexa. Fiuza e Sarriera (2013) avaliam que a permanência difere da persistência, enquanto aquela envolve diferentes formas de avaliação adotadas pela própria instituição, esta última é apenas o resultado da decisão do estudante em continuar participando das atividades pedagógicas naquele curso ou instituição. De uma maneira sintética, o comportamento de persistência no curso gera a permanência (Fiuza \& Sarriera, 2013) e, com isso, evita-se a evasão. Apesar de permanência não ser sinônimo de persistência refletem o mesmo comportamento, no primeiro caso reflete a visão do estudante, no segundo, da instituição (Fiuza \& Sarriera, 2013). No presente estudo serão tomados como apenas um comportamento, definido como permanência, em linha com a maioria dos estudos nacionais sobre o tema (Santos Júnior \& Real, 2017). A evasão, contudo, refere-se a um conceito diferente, fomentar a permanência pode reduzir a evasão, mas conceitualmente referem-se a fenômenos diferentes (Fiuza \& Sarriera, 2013).

Existe uma série de modelos teóricos sobre o comportamento de evasão e permanência no ensino superior (Costa \& Gouveia, 2018). Estudos baseados inicialmente na teoria do suicídio (Durkheim, 2000), como o de Spady, propuseram que as condições de permanência de um estudante na universidade, e qualidade dessas condições, dependem da sua integração ao tecido social. O modelo de Tinto (1993), talvez seja um dos mais citados, chamado de Teoria da Integração do Estudante, considera como variáveis relevantes no processo a integração acadêmica, o compromisso com o objetivo e com a instituição, qualidade do esforço do estudante e compromissos externos. O modelo de Processo do Abandono de Spady (1970) é bastante próximo ao de Tinto, mas inclui varáveis de cunho mais sociológico (Costa \& Gouveia, 2018), como o contexto familiar. Modelos mais recentes, como o Modelo Integrado de Permanência de Cabrera, Nora e Castañeda (1992), e o Modelo do Comprometimento Estudante-Instituição de Nora, Barlow e Crisp (2005) também se baseiam em variáveis semelhantes.

Nesse sentido, Davidson, Beck \& Milligan (2009) desenvolveram o College Persistence Questionnaire (CPQ), um instrumento capaz de avaliar a maioria das dimensões relevantes no processo e incluídas em estudos anteriores. A expressão "college persistence" do instrumento original foi traduzida como permanência acadêmica a fim de manter seu sentido original, uma vez que a tradução literal, algo como persistência na faculdade, não possibilitaria isso. Assim, a versão brasileira do instrumento foi chamada de Questionário de Permanência Acadêmica (QPA), em acordo com as expressões mais utilizadas nas pesquisas sobre o tema (Santos Júnior \& Real, 2017).

O estudo original dos autores identificou seis dimensões: comprometimento institucional, comprometimento com a graduação, integração acadêmica, integração social, satisfação com os serviços de apoio e personalidade e ajustamento (Davidson et al., 2009). As dimensões de comprometimento institucional e comprometimento com a graduação desempenham um papel crucial nos modelos causais contemporâneos de permanência e parecem exercer efeitos diretos sobre as decisões de persistência (Costa \& Gouveia, 2018). O comprometimento institucional relaciona-se com a percepção que o estudante tem da instituição, já o comprometimento com a graduação relaciona-se diretamente com a possibilidade de obtenção do diploma (Costa \& Gouveia, 2018). As dimensões de integração social e integração acadêmica partem da teoria de Tinto (1993) e postulam que o ajuste bem-sucedido dos alunos passa pelas etapas de separação, transição e integração nos tecidos acadêmicos e sociais da vida universitária. A integração social é relacionada ao envolvimento dos estudantes em atividades socias no campus (Costa \& Gouveia, 2018). A integração acadêmica remete à habilidade do estudante de participar ativamente do ambiente acadêmico da instituição, em atividades, tais como relação com docentes, participação em grupos de estudo e horas dedicadas ao estudo, (Costa \& Gouveia, 2018). A satisfação com os serviços de apoio foi identificada por Davidson et al. (2009) como um tema presente já nas primeiras pesquisas sobre o assunto (Davidson et al., 2009). O CPQ incluiu diversos itens relativos aos serviços institucionais de apoio, os quais, em estudos anteriores, foram associados ao comportamento de permanência (Davidson et al., 2009). Uma última dimensão relacionava a personalidade e variáveis de ajuste à permanência, trata-se da dimensão de personalidade e ajustamento. Existem evidências em estudos brasileiros que associam estas e outras variáveis relativas a diferenças individuais 
à persistência dos estudantes (Costa \& Gouveia, 2018). Após análises iniciais, Davidson et al. (2009) diminuíram essa dimensão para três itens e renomearam como Consciência Acadêmica.

As dimensões comprometimento institucional, integração acadêmica e consciência acadêmica foram bons preditores diretos do comportamento de permanência (Davidson et al., 2009). As demais dimensões do CPQ afetam as decisões de persistência de modo indireto, contudo, a nível individual, são bons indicadores de situações de risco de permanência (Pugh, Cramer, Slatyer, Twigg, \& Robinson, 2018). Alguns modelos teóricos sobre o tema propõem estruturas conceituais integrando essas variáveis (e.g., Cabrera et al., 1992; Davidson, Beck, \& Grisaffe, 2015).

Existem outros instrumentos semelhantes contruídos no contexto brasileiro (Almeida et al., 2019; Ambiel, 2015), apesar de sua boa qualidade psicométrica e estrutura bastante semelhante, seu foco recai sobre o abandono ou evasão, e não especificamente sobre a permanência. O CPQ se torna um instrumento relevante para ser trazido ao contexto brasileiro por abordar a questão a partir da permanência na universidade.

Costa et al. (2018) alertam que, no contexto brasileiro, os estudos sobre o tema tendem a focar na perspectiva da gestão universitária. Por outro lado, as questões relativas à permanência do estudante sob a ótica deste centram-se no processo de adaptação à universidade (Mata et al., 2017). O processo de adaptação é sem dúvida relevante na temática da permanência, mas a diversidade de modelos disponíveis aponta para outras dimensões de grande impacto, sobretudo a sociológica (Costa \& Gouveia, 2018). Nesse sentido, o questionário desenvolvido por Davidson et al. (2009), ao abordar diversas dimensões, traria alguma inovação e seria sem dúvida uma contribuição para estudos sobre o tema.

Com o intuito de abarcar a crescente demanda por melhorar as condições de permanência na universidade, sobretudo do estudante em vulnerabilidade socioeconômica (Imperatori, 2017), algumas políticas específicas foram desenvolvidas no setor público, com destaque para o Programa Nacional de Assistência Estudantil (PNAES), formulado em 2008. Essa política, lançada como programa, unificou uma série de esforços pontuais de apoio à permanência desenvolvidos, em alguns casos, há décadas (Imperarori, 2017). O PNAES foi criado com objetivo de ampliar as condições de acesso e permanência à educação superior, minimizar os efeitos das desigualdades sociais e regionais, bem como promover inclusão social pela educação superior, e reduzir as taxas de retenção e de evasão (Imperatori, 2017; Santos Júnior \& Real, 2017). Apesar desses esforços ainda existem ressalvas quanto à efetividade dessas políticas (Paula, 2017). As ações de assistência estudantil são organizadas em áreas temáticas, como moradia estudantil, alimentação transporte e apoio pedagógico. Alguns estudos destacam que estratégias com foco na alimentação e moradia são mais relevantes (Lacerda \& Valentini, 2018; Morosini et al., 2011). Mas os resultados ainda não são conclusivos sobre como atuam as ações de assistência estudantil, a nível individual, sobre o comportamento de permanência na universidade e diminuição da evasão (Andrade \& Teixeira, 2017). Nesse sentido, são necessários mais estudos que identifiquem a efetividade dessas ações em promover a permanência e quais ações vinculadas à assistência estudantil teriam efeito sobre o comportamento de permanência e evasão.

\section{Método}

\section{Participantes}

Participaram desse estudo 339 estudantes de graduação de uma universidade pública federal do sul do país, $203(59,9 \%)$ do sexo feminino, $126(37,2 \%)$ do sexo masculino, 10 (3\%) preferiram não declarar ou informaram outro sexo. A faixa etária foi de 18 a 56 anos e a média foi de 23,37 ( $D P=5,71)$. Quanto a áreas de seus cursos, 29,4\% estavam inscritos em curso da área de Ciência Humanas e Sociais Aplicada; 20,1\% em ciências da Saúde; 19,4\% em Engenharias; 12,2\% na área de Linguística, Letras e Artes; $11 \%$ na área de Agrárias, 7,9\% na área de Ciências Exatas e da Terra. Quanto ao tempo de universidade, $20,9 \%$ estavam de um a quatro semestres na universidade; $38,6 \%$ de cinco a oito semestres e $40,56 \%$ já estavam a mais de oito semestres na universidade. Todos os participantes possuíam acesso a algum dos benefícios oferecidos no âmbito da política nacional de assistência estudantil (i.e., moradia, alimentação, transporte, atenção odontológica, bolsa formação, auxílio material pedagógico, apoio psicológico, apoio pedagógico, apoio psicossocial).

\section{Instrumentos}

Questões sociodemográficas. Constituído de questões sobre idade, estado civil, cidade de origem, ano de ingresso na universidade e curso frequentado. Continham também questões sobre troca de curso.

Questões sobre acesso às ações de assistência estudantil. Um questionário de sete perguntas, sendo a maioria dicotômicas (sim/não) sobre o conhecimento dos estudantes à respeito dos serviços oferecidos pela assistência estudantil da universidade pesquisada e uma questão sobre quais desses serviços o estudante utiliza (e.g., "Você conhece os serviços de apoio social, emocional e pedagógico que são oferecidos aos estudantes da sua universidade"). Essas questões foram elaboradas com base no conhecimento prático de dois técnicos administrativos em educação com experiência de trabalho em assistência estudantil e constituídas a partir de questionários já usados internamente pelas unidades da universidade onde atuam.

Questionário de permanência acadêmica (College Permanence Questionnaire, Davidson et al., 
2009). O questionário é constituído de 36 itens divididos em seis subescalas ou dimensões: compromisso institucional (Institutional commitment,), com quatro itens (e.g., "O quão confiante vocês está que esta é a universidade certa para você?"); compromisso com a diplomação (Degree Commitment), com seis itens (e.g., "Neste momento, o quão forte é seu compromisso com obter o diploma, aqui ou em outro lugar?"); integração social (Social Integration), de oito itens (e.g., "Qual é a sua impressão geral sobre os outros alunos?"); integração acadêmica (Academic Integration), de oito itens (e.g., "Em geral, quanto você está satisfeito com a qualidade do ensino que está recebendo aqui?”); apoio institucional (Support Services Satisfaction) de seis itens (e.g., "Como você classificaria o apoio acadêmico que recebe aqui?") e consciência acadêmica (Academic Conscientiousness) de quatro itens (e.g., "Com que frequência você entrega trabalhos com atraso?"). Todas as respostas foram dadas em uma escala tipo Likert de cinco pontos. No entanto, cada subescala recebe um tipo de resposta, por exemplo, na questão "O quão confiante você está que esta é a universidade certa para você", utiliza-se uma escala de confiança, 1 equivale a "Nenhuma confiança" e 5 a "Muitíssima confiança". Já na questão "Qual é a sua impressão geral sobre os outros alunos?", utiliza-se uma escala de favorabilidade, 1 equivale a "Nada favorável" e 5 a "Muito favorável". Apenas a subescala de consciência acadêmica possui codificação reversa. A versão original da escala produziu coeficientes alfa de Cronbach de 0,63 a 0,82 (Davidson, et al., 2009).

\section{Procedimentos}

Após obter aceitação dos autores do questionário, procedeu-se à adaptação do QPA para o português do Brasil seguindo as etapas orientadas de tradução, síntese, retrotradução, comitê de especialistas e pré-teste. Primeiramente, foi realizado o processo de tradução do idioma de origem, inglês, para o português do Brasil, por dois tradutores bilíngues. Após isso, foi realizada a comparação das versões, observando equivalência semântica, idiomática e conceitual, para ser realizada a síntese das versões, o que se refere à segunda etapa da adaptação. A seguir, foi realizada uma retrotradução conduzida por profissional bilíngue, fluentes em inglês e falante nativo de português e familiarizado com o construto. Após discussões entre os pesquisadores e tradutor, essa etapa confirmou que todos os itens eram equivalentes à versão em inglês. Logo, a escala foi analisada por especialistas com familiaridade com os construtos, que realizaram julgamento sobre a pertinência técnica e teórica dos itens elaborados. Todos concordaram que os itens traduzidos eram adequados para o estudo. Os especialistas sugeriram algumas mudanças, nomeadamente: trocar a expressão "diplomação" para "concluir a graduação" no item 4 e 5 da dimensão Compromisso com a diplomação. Também foi sugerida a substituição em todas as questões com o item "nesta universidade" pelo nome da universidade pesquisada. Nessa etapa, não foram sugeridas novas alterações. Na quarta etapa do processo de adaptação, o instrumento adaptado foi submetido à avaliação do público-alvo, composto de estudantes de graduação, para avaliação da compreensão dos itens. Foi distribuído o link para preenchimento on-line do questionário para sete estudantes universitários. Nesse convite, foi pedido que os estudantes opinassem sobre os itens. Todos os participantes consideraram os itens compreensíveis e adequados para a avaliação. Para a coleta de dados, foi utilizada a plataforma SurveyMonkey (www.surveymonkey.com) que foi configurada juntamente com o consentimento avisado, as informações sociodemográficas e culturais e os instrumentos utilizados.

\section{Procedimentos Éticos}

Foram tomados todos os cuidados para a realização de pesquisas com seres humanos, em acordo com a Resolução no 196 de 1996, do Conselho Nacional de Saúde. O projeto foi aprovado pelo Comitê de Ética para Pesquisas da universidade em que foi realizada a coleta de dados (CAAE: 01478318.0.0000.5346).

\section{Procedimento de Análise dos Dados}

As análises foram realizadas utilizando-se o programa Statistical Package for the Social Sciences, versão 20 (IBM SPSS Statistics 20, SPSS Inc., Chicago, IL) e o pacote estatístico AMOS versão 21.0. O método de distância de Mahalanobis foi utilizado; nenhum caso apresentou distribuições multivariadas. A assimetria entre as variáveis não foi superior a $3(-1,86$ a 1,28$)$ e a curtose não ultrapassou $7(-0,86$ a 2,88), não sendo, portanto, necessárias remoções (Kline, 2016).

Dado que a estrutura do QPA foi apoiada em pesquisas anteriores (Beck \& Milligan, 2014; Betts, Shirley, \& Kennedy, 2017; Davidson et al., 2009; MéndezGiménez, Fernandez-Rio, \& Cecchini, 2014; Pugh et al., 2018) apenas a análise fatorial confirmatória (AFC) foi realizada (Kline, 2016). Diferentes modelos fatoriais, medidas e modelos estruturais foram testados $\mathrm{e}$ comparados, em primeiro lugar, por meio da relação qui-quadrado/graus de liberdade $\left(\chi^{2} / g l\right)$. Valores maiores que 2 indicam um ajuste inadequado (Byrne, 2010). Também foram empregados os seguintes índices de ajuste de qualidade: Erro Quadrático Médio de Aproximação (RMSEA) (Hu \& Bentler, 1999) e Raiz Média Quadrada Residual (RMR) para os quais pontuações menores que 0,05 significam excelente ajuste e escores entre 0,05 e 0,8 significa bom ajuste (Hu \& Bentler, 1999) e o Índice de Ajuste Comparativo (CFI; Hu \& Bentler, 1999), para o qual escores acima de 0,90 significam bom ajuste. Outro critério, o Critério de Informação de Akaike (AIC) foi usado na comparação de modelos, com valores menores indicando um melhor ajuste do modelo (Jiang, 2017). As análises relativas aos serviços de assistência estudantil foram realizadas por meio de testes de diferenças de 
médias (Teste $t$ de Student, Anova) tendo sido observados e cumpridos os pré-requisitos de independência das observações normalidade e homogeneidade das variâncias (Field, 2018).

\section{Resultados}

\section{Análise Fatorial Confirmatória (AFC) uma Confiabilidade}

Com o intuito de atenuar problemas comuns em análises de instrumentos com um número elevado de itens, optou-se por agrupar os itens em parcelas (item parceling) (Hau \& Marsh, 2004). Contudo, a redução dos itens deve basear-se na presunção teórica e empírica da unidimensionalidade de cada escala e seguir critério de agrupamento específicos (Little, Cunningham, Shahar, \& Widaman, 2002).

A presunção da unidimensionalidade foi testada no teste de scree plots, eigenvalues maiores que 1.0., itens com um coeficiente mínimo de .40 e interpretação dos fatores (Tabachnick \& Fidell, 2001). Para isso, os fatores foram extraídos pelo método dos Principais Eixos Fatoriais, com rotação ortogonal (assumindo que os fatores extraídos são independentes uns dos outros) por intermédio do método Varimax. Nessa etapa, identificou-se que duas dimensões apresentavam estrutura bidimensional, integração social e compromisso com a diplomação. $\mathrm{Na}$ dimensão integração social, o item 7 mostrou carga diferentes dos demais, na dimensão Compromisso com a diplomação, o item 2 teve esse mesmo comportamento. Uma nova análise de dimensionalidade sem esses itens revelou uma estrutura unidimensional.

Cumprida a presunção empírica da unidimensionalidade, o próximo passo foi elaborar as parcelas. Essa etapa foi realizada com base na recomendação de que cada construto tivesse ao menos três itens (Klein, 2006; Little et al., 2002). Integração acadêmica, integração social, compromisso com a diplomação, apoio institucional e compromisso institucional foram representados por três parcelas com 1-3 itens por parcela (A consciência acadêmica, com quatro itens, não teve itens parcelados).

É recomendado que modelos alternativos sejam testados, especialmente dado ao tamanho do modelo de medida (Byrne, 2010). Para contrastar o modelo de medida com as variáveis de integração social, integração acadêmica, apoio institucional e compromisso com a diplomação organizado em parcelas (Modelo 1) foram propostas mais duas possibilidades. O Modelo 2 dividiu em parcelas apenas as variáveis de integração social e integração acadêmica, visto serem as que contaram com maior número de itens, ainda que não exista uma referência clara sobre a partir de quantos itens recomenda-se o parcelamento (Little et al., 2002). O Modelo 3 utilizou a mesma estrutura do anterior, mas removeu os itens 2 e 7 da dimensão Compromisso com a diplomação em razão dos achados da análise de dimensionalidade.

Tabela 1

Sumário dos Índices de Ajustes da Análise Fatorial Confirmatória do QPA $(n=339)$

\begin{tabular}{|c|c|c|c|c|c|c|}
\hline Modelo & $\left(\chi^{2} / g l\right)$ & gl & CFI & RMSEA $(90 \%$ CI $)$ & SRMR & AIC \\
\hline Modelo 1 & 3,71 & 155 & 0,85 & 0,09 & 0,068 & 685,81 \\
\hline Modelo 2 & 3,29 & 260 & 0,81 & 0,08 & 0,074 & 986,937 \\
\hline Modelo 3 & 3,26 & 260 & 0,81 & 0,08 & 0,071 & 979,251 \\
\hline
\end{tabular}

Nota. CFI=Índice de Ajuste Comparativo; RMSEA=Erro Quadrático Médio de Aproximação; SRMR=Raiz Média Quadrada Residual; AIC=Critério de Informação de Akaike

Ao comparar os modelos (Tabela 1), foi escolhido o Modelo 3 que apresentou melhor ajuste de modelo: $\quad \chi^{2}(237)=803,43, \quad \mathrm{CFI}=0,81, \quad \mathrm{SRMR}=0,07$, RMSEA $=0,08$ e AIC $=928,94$. O Modelo 1 apesentou valores altos para o RMSEA, já os Modelos 2 e 3 mostram valores de ajuste melhores nesse índice e muito semelhante nos demais. Embora os modelos mostrem quase a mesma relação $\chi^{2} / g l$, não há acordo universal sobre esse indicador (Kline, 2016). No entanto, o menor valor de AIC do Modelo 3 (em comparação com o Modelo 2) indicou um melhor ajuste para esse modelo. Uma anotação deve ser feita sobre o valor do CFI, cujo valor identificado está abaixo dos 0,90 recomendados ( $\mathrm{Hu} \&$ Bentler, 1999). No contexto desse estudo, de caráter confirmatório, o valor do CFI não é considerado problemático
(Rigdon, 1996), sendo mais relevante o RMSEA, o qual nesse caso está dentro dos limites esperados para um bom ajuste $(0,05$ e 0,08, Hu \& Bentler, 1999).

A estatística descritiva e matriz de correlações de Pearson, a média, desvio padrão e a matriz de correlações para cada uma das quatro subescalas do QPA aparecem na Tabela 2. Todas as subescalas se correlacionam significativamente $(p<0,01)$.

Adicionalmente, foram realizados testes de consistência interna por meio do coeficiente alfa de Cronbach e comparado com os obtidos por Davidson et al. (2009, 2015), esta última foi uma versão reduzida de 32 itens, representada na Tabela 3. A semelhança dos coeficientes obtidos com a versão brasileira e o estudo original colabora para suportar a fiabilidade do instrumento. 
Tabela 2

Médias, Desvios Padrões e Coeficientes de Correlação entre as Subescalas do QPA

\begin{tabular}{|c|c|c|c|c|c|c|c|c|}
\hline Variável & 1 & 2 & 3 & 4 & 5 & 6 & $\mathrm{M}$ & $\mathrm{DP}$ \\
\hline 1. Integração social & - & & & & & & 3,58 & 0,59 \\
\hline 2. Integração acadêmica & $0,41^{* *}$ & - & & & & & 3,52 & 0,66 \\
\hline 3. Compromisso c/ diplomação & $0,38^{* *}$ & $0,60^{* *}$ & - & & & & 4,17 & 0,66 \\
\hline 4. Apoio institucional & $0,37^{* *}$ & $0,65^{* *}$ & $0,53^{* *}$ & - & & & 3,24 & 0,64 \\
\hline 5. Consciência acadêmica & $0,14^{* *}$ & $0,38^{* *}$ & $0,39 * *$ & $0,35^{* *}$ & - & & 4,15 & 0,90 \\
\hline 6. Compromisso institucional & $0,39^{* *}$ & $0,56^{* *}$ & $0,68^{* *}$ & $0,49^{* *}$ & $0,40^{* *}$ & - & 4,15 & 0,77 \\
\hline
\end{tabular}

Nota. ${ }^{* *} p<0,01$

Tabela 3

Coeficientes de Alfa a de Cronbach da Amostra Brasileira em Comparação com Davidson et al. $(2009,2015)$

\begin{tabular}{lccc}
\hline \multicolumn{1}{c}{ Subescala } & Amostra brasileira & Davidson et al. (2009) & Davidson et al. (2015) \\
\hline Integração acadêmica & 0,82 & 0,81 & 0,83 \\
Integração social & 0,73 & 0,82 & 0,80 \\
Compromisso com a diplomação & 0,72 & 0,70 & 0,70 \\
Apoio institucional & 0,71 & 0,74 & 0,78 \\
Consciência acadêmica & 0,75 & 0,63 & 0,68 \\
Compromisso institucional & 0,68 & 0,78 & 0,83 \\
\hline
\end{tabular}

\section{Interação das Dimensões do QPA com as Ações de Assistência Estudantil}

As questões sobre os serviços de assistência estudantil oferecidos e acessados pelos estudantes e seu impacto sobre a permanência e a evasão foi o próximo passo de análise. Estas foram realizadas com todas as escalas do QPA, entretanto, sabe-se que elas refletem diferentes componentes do comportamento em questão (Davidson et al., 2009). A primeira análise empreendida foi das diferenças entre as pessoas que declararam já terem utilizado serviços de apoio social, emocional e pedagógico da universidade e aquelas declararam não terem acessado esses serviços. Testes $t$ de Student encontraram diferenças significas em todas as subescalas do QPA entre estudantes que relataram acessar e não acessar esses serviços de assistência. Os resultados estão apresentados na Tabela 4.
Nota-se que todas as diferenças de médias são em favor das pessoas que não acessaram os serviços, isto é, aquelas que não utilizaram os serviços de apoio à permanência da universidade obtiveram pontuações mais altas em todas as subescalas do QPA. Com exceção apenas da subescala de Compromisso institucional, que não mostrou diferenças significativas.

Quanto aos obstáculos para acessar os serviços de apoio, houve também diferenças estatisticamente significativas nas subescalas do QPA em relação aos estudantes que relataram alguma dificuldade para acessar aos serviços de apoio à permanência oferecidos pela universidade e os que não relataram dificuldades (Tabela 5). A análise das médias indica que aqueles estudantes que relataram dificuldades em acessar a esses serviços tiveram menor pontuação em todas as subescalas do QPA.

Tabela 4

Teste de Diferenças nas Subescalas do QPA em Relação ao Acesso a Serviços de Apoio

\begin{tabular}{|c|c|c|c|c|}
\hline \multirow{3}{*}{ Subescalas QPA } & \multicolumn{4}{|c|}{ Já utilizou algum serviço de apoio social, emocional ou pedagógico } \\
\hline & Sim & Não & \multirow{2}{*}{$t$} & \multirow[b]{2}{*}{$p$} \\
\hline & $\mathrm{M}(\mathrm{DP})$ & $M(D P)$ & & \\
\hline Integração social & $3,51(0,65)$ & $3,64(0,54)$ & 1,99 & * \\
\hline Integração acadêmica & $3,42(0,67)$ & $3,59(0,65)$ & 2,4 & * \\
\hline Compromisso c/ diplomação & $4,07(0,68)$ & $4,25(0,64)$ & 2,43 & * \\
\hline Apoio institucional & $3,14(0,67)$ & $3,31(0,61)$ & 2,47 & * \\
\hline Consciência acadêmica & $3,97(0,95)$ & $4,29(0,84)$ & 3,32 & $* *$ \\
\hline Compromisso institucional & $4,06(0,75)$ & $4,22(0,77)$ & 1,94 & 0,053 \\
\hline
\end{tabular}

Nota. ${ }^{*} p<0,05 ;{ }^{* *} p<0,01$ 
Tabela 5

Teste de Diferenças nas Subescalas do QPA em Relação à Percepção de Obstáculos ao Acesso a Serviços de Apoio

\begin{tabular}{|c|c|c|c|c|}
\hline \multirow{3}{*}{ Subescalas QPA } & \multicolumn{4}{|c|}{ Vê empecilhos em procurar os serviços de apoio à permanência? } \\
\hline & $\operatorname{Sim}$ & Não & \multirow{2}{*}{$t$} & \multirow[b]{2}{*}{$p$} \\
\hline & $\mathrm{M}(\mathrm{DP})$ & $\mathrm{M}(\mathrm{DP})$ & & \\
\hline Integração social & $3,41(0,64)$ & $3,67(0,54)$ & 3,96 & $* * *$ \\
\hline Integração acadêmica & $3,26(0,62)$ & $3,67(0,64)$ & 5,6 & *** \\
\hline Compromisso c/ diplomação & $3,93(0,73)$ & $4,31(0,59)$ & 5,15 & *** \\
\hline Apoio institucional & $2,89(0,59)$ & $3,43(0,59)$ & 7,88 & *** \\
\hline Consciência acadêmica & $3,85(0,92)$ & $4,31(0,85)$ & 4,49 & *** \\
\hline Compromisso institucional & $3,95(0,82)$ & $4,27(0,71)$ & 3,65 & $* * *$ \\
\hline
\end{tabular}

Nota. ${ }^{* * *} p<0,001$

Uma última verificação foi feita relativamente aos serviços acessados, foram elencados os serviços de assistência estudantil oferecidos pela universidade e pedido que o estudante indicasse qual ou quais serviços fazia uso. Notase que o serviço mais utilizado pelo estudante participante da pesquisa é o restaurante universitário (95,9\%), seguido da moradia estudantil (55,2\%). Apenas 20\% $(n=68)$ dos estudantes utilizam apenas um serviço; 48,8\% $(n=166)$ utilizam dois ou três serviços; $28,6 \%(n=97)$ utilizam mais de quatro serviços, até um máximo de sete. Contudo, realizada uma Análise de Variância (ANOVA), não foram encontradas diferenças estatisticamente significativas entre os estudantes que acessaram um, de dois a três e mais de quatro serviços entre as escalas do QPA.

Dentro dessa linha de análise e entendendo que os serviços diferem qualitativamente (p. ex., o acesso moradia estudantil poderá ter mais impacto que o apoio odontológico), os serviços foram explorados de três maneiras diferentes. Primeiramente foram analisadas diferenças entre aqueles estudantes que recebiam algum tipo de apoio pecuniário (bolsas) e os que não recebiam. Depois, os estudantes que recebiam algum tipo de apoio social, psicossocial ou odontológico e os que não recebiam. Por último foram analisadas diferenças entre estudantes que faziam uso e não faziam uso da moradia estudantil oferecida pela universidade. Todas as diferenças foram avaliadas com base em Testes t, nenhuma análise revelou diferenças estatisticamente significativas. Nessa amostra de estudantes, nem o acesso a bolsas, nem os serviços de apoio psicossocial e à saúde e nem a moradia estudantil mostrou diferenças em relação às subescalas do QPA.

\section{Discussão}

A identificação dos fatores envolvidos no processo da permanência e abandono da universidade é crucial no desenvolvimento da universidade no Brasil e na garantia do acesso democrático a essa universidade (Imperatori, 2017; Santos Júnior \& Real, 2017). Trabalhos como de Ambiel (2015) e Almeida et al. (2019) são um interessante contributo, contudo seu foco recai sobre a evasão. Também, não foram encontrados instrumentos adaptados ao contexto brasileiro com o objetivo de avaliar esses fatores na perspectiva da permanência no ensino superior. O presente estudo teve por objetivo adaptar à realidade brasileira e buscar evidência de validade de uma escala capaz de avaliar as principais dimensões relativas à permanência no ensino superior. Para esse fim, foi utilizado o instrumento de Davidson et al. (2009), construído em consonância com os enquadramentos teóricos mais reconhecidos da área (e.g., Spady, 1970; Tinto, 1993).

A versão original do instrumento de Davidson et al. (2009), chamado aqui de QPA, com 36 itens divididos em seis dimensões (subescalas), foi testada por meio de análises fatoriais confirmatória em três diferentes modelos apresentados a fim de explorar diferentes composições (Byrne, 2010). As duas primeiras utilizaram diferentes formas de parcelamento de itens (Hau \& Marsh, 2004). A terceira versão foi apresentada sem dois itens que com base nas análises de dimensionalidade realizada na etapa de parcelamento (Little et al., 2002) poderiam representar problemas de ajuste do modelo de medida. Essa última estrutura foi a que obteve melhores índices de ajuste.

A correlação entre as subescalas do QPA mostrou significância estatística em todos relacionamentos. As relações envolvendo compromisso com a diplomação, apoio institucional e integração acadêmica (todas com $r>0,60$ ) seguem a linha de estudos anteriores (Beck, Milligan, Lindheimer, \& Osborn, 2012; Davidson et al., 2009). Os coeficientes de confiabilidade (alfa de Cronbach) são bastante próximos da versão original, com 36 itens (Davidson et al., 2009) e de uma mais atual com 32 itens (Beck et al., 2012).

O segundo objetivo do estudo foi identificar como as ações de assistência estudantil efetuadas pela universidade estudada atuam sobre as dimensões relativas à permanência e evasão no ensino superior avaliadas pelo QPA. Para tanto, utilizou-se um questionário com diversas perguntas sobre o acesso aos serviços de assistência estudantil oferecidos pela universidade. A primeira análise sobre as diferenças entre os estudantes que já haviam acessado algum tipo de apoio social, emocional ou pedagógico na universidade revelou um pior desempenho em quase todas as 
subescalas do QPA em relação aos que não haviam utilizado os mesmos serviços. Os resultados à primeira vista são contra intuitivos, mas do ponto de vista lógico mostra que os serviços estão sendo acessados precisamente pelo estudante que dele precisa. O fato de apenas a escala de compromisso institucional não ter mostrado diferenças mostra que apesar da situação de risco à permanência que se encontram os estudantes, eles não diferem em nada dos demais em relação à confiança e satisfação com sua universidade, talvez por isso tenham procurado os serviços

Semelhante a essa questão de pesquisa, a próxima análise revelou que as pessoas que relataram dificuldades em acessar os serviços de assistência estudantil oferecidos pela universidade tiveram uma média mais baixa em todas as escalas. Os resultados indicam claramente que, mesmo que as pessoas possam acessar os serviços, algumas ainda observam barreiras no seu acesso, mostrando que, para além da oferta dos serviços de assistência, é necessário identificar que barreiras existem. Como já advertiu Paula (2017), as dificuldades são de ordem simbólico-subjetiva, criando um processo de exclusão no interior das universidades que não se adapta ao estudante em vulnerabilidade socioeconômica que busca as ações de assistência estudantil.

Ainda dentro dos objetivos do estudo, tratou-se de identificar qual a colaboração das ações organizadas em grupo (pecuniário, apoio psicossocial e à saúde e moradia) sobre os escores no QPA. Os resultados mostraram não haverem diferenças estatisticamente significativas em relação às subescalas do QPA, diferente do que seria esperado (Lacerda \& Valentini, 2018; Morosini, 2011). Resultados semelhantes foram encontrados por Andrade e Teixeira (2017). Nesse estudo, os autores encontraram correlações muito baixas do acesso aos serviços com os indicadores de permanência. Os autores atribuem os resultados à falta de diretrizes e indicadores concretos que possam dirigir e avaliar as políticas de assistência estudantil.

Os resultados encontrados sobre as diferenças entre os estudantes que acessaram os serviços de apoio psicossocial e à saúde (odontologia e psicologia) da universidade estudada mostram que são um fator de apoio ao estudante em dificuldades. Tendo em vista que as dificuldades não são apenas de ordem financeira, mas simbólicas, pedagógicas e relacionais (Paula, 2017), os serviços devem se manter abertos e próximos ao estudante que deles necessite, utilizando todas as estratégias de apoio possíveis. Apesar dos resultados, pode se dizer que as ações estão no caminho certo. Ações como alimentação e moradia ainda são cruciais, tanto é, que não foi possível comparar os estudantes que acessavam e os que não acessavam o apoio alimentação, pois 95,9\% dos estudantes na amostra acessaram esse serviço. Quanto à moradia, sabe-se que nessa universidade possivelmente todos que dela necessitam acessam o apoio específico (Vautero, Pozzobon, \& Zanoello, 2009), não sendo possível uma comparação. $\mathrm{O}$ diferencial no aumento das condições e permanência e redução da evasão, no que tange à política de assistência estudantil, parece estar precisamente nos serviços de atenção psicossocial, pedagógica e saúde. Resta monitorar e avaliar melhor esses serviços, QPA se coloca como uma ferramenta padrão de avaliação, para fornecer orientação confiável quanto à efetividade do programa, guiar programas e alocação de recursos (Beck \& Davidson, 2010).

\section{Limitações e Estudos Futuros}

Algumas limitações podem ser indicadas, a primeira de refere à amostra, dados os objetivos do estudo foram incluídos apenas estudante que acessavam as políticas de assistência estudantil de forma extensiva, nessa universidade chamado de benefício socioeconômico (Vautero et al., 2009). Um trabalho futuro poderia avaliar as subescalas do QPA no universo geral da universidade e identificar quais fatores poderiam pôr em risco sua permanência na universidade. Um outro ponto de limitação se dá em relação ao acesso aos serviços. Na universidade estudada, havia uma limitação de acesso a outros tipos de apoio, como a saúde, visto ser oferecido apenas dois tipos (serviço de odontologia e apoio psicológico). Estudos anteriores já identificaram o peso desse tipo de apoio (Andrade \& Teixeira, 2017). Recomenda-se que sejam estudadas instituições com uma gama de serviços maiores e compará-las com outras para que seja possível avaliar quais programa teriam um efeito maior sobre a permanência.

\section{Agradecimentos}

Não há menções.

\section{Financiamento}

A presente pesquisa não recebeu nenhuma fonte de financiamento sendo custeada com recursos dos próprios autores.

\section{Contribuições dos autores}

Declaramos que todos os autores participaram da elaboração do manuscrito. Especificamente, os autores Jaisso Vautero e Luciane Pozobon participaram da redação inicial do estudo - conceitualização, investigação, visualização e análise dos dados, e a autora Ana Daniela Silva participou da redação final do trabalho - revisão e edição.

\section{Disponibilidade dos dados e materiais}

Todos os dados e sintaxes gerados e analisados durante esta pesquisa serão tratados com total sigilo devido às exigências do Comitê de Ética em Pesquisa em Seres Humanos. Porém, o conjunto de dados e sintaxes que apoiam as conclusões deste artigo estão disponíveis mediante razoável solicitação ao autor principal do estudo.

\section{Conflito de interesses}

Os autores declaram que não há conflitos de interesses. 


\section{Referências}

Almeida, L. S., Casanova, J. R., Bernardo, A. B., Cervero, A., Santos, A. A., \& Ambiel, R. A. M. (2019). Construção de um questionário transcultural de motivos de abandono do ensino superior. Avaliação Psicológica, 18(2), 201-209. doi: 10.15689/ap.2019.1802.17694.11

Ambiel, R. A. M. (2015). Construção da Escala de Motivos para Evasão do Ensino Superior. Avaliação Psicológica, 14(1), 41-52. Recuperado de https://www.redalyc.org/pdf/3350/335042985006.pdf

Andrade, A. M. J., \& Teixeira, M. A. P. (2017). Áreas da política de assistência estudantil: Relação com desempenho acadêmico, permanência e desenvolvimento psicossocial de universitários. Avaliação: Revista da Avaliação da Educação Superior (Campinas), 22(2), 512-528. doi: $10.1590 / \mathrm{s} 1414-40772017000200014$

Beck, H. P., \& Davidson, W. B. (2010). College Persistence Questionnaire. https://www.beckdavidson.com/welcome/welcome.aspx

Beck, H. P., \& Milligan, M. (2014). Factors influencing the institutional commitment of online students. Internet High. Educ. 20, 51-56. doi: 10.1016/j.iheduc.2013.09.002

Beck, H. P., Milligan, M., Lindheimer, J., \& Osborn, M. (2012). The College Persistence Questionnaire: Developing scales to assess student retention. Trabalho apresentado no encontro da Southern Society for Philosophy and Psychology, Savannah, GA.

Betts, K. J., Shirley, J. A., \& Kennedy, R. (2017). Nursing Students Using the College Persistence Questionnaire. Journal of Education and Practice, 8(12). Recuperado de https://files.eric.ed.gov/fulltext/EJ1140641.pdf

Byrne, B. M. (2010). Structural equation modeling with AMOS: Basic concepts, applications, and programming (2a ed.). New York: Routledge.

Cabrera, A. F., Nora, A., \& Castañeda, M. B. (1992). The role of finances in the persistence process: A structural model. Research in Higher Education, 33(5), 571-593. doi: 10.1007/BF00973759

Costa, F. J. C., Bispo, M. S, \& Pereira, R. C. F. (2018). Dropout and retention of undergraduate students in management: A study at a Brazilian Federal University. RAUSP Management Journal, 53(1), 74-85. doi:1016/j.rauspm.2017.12.007

Costa, O. S., \& Gouveia, L. B. (2018). Modelos de retenção de estudantes: Abordagens e perspectivas. REAd. Revista Eletrônica de Administração (Porto Alegre), 24(3), 155-182. doi: 10.1590/1413-2311.226.85489

Davidson, W. B., Beck, H. P., \& Grisaffe, D. B. (2015). Increasing the Institutional Commitment of College Students: Enhanced Measurement and Test of a Nomological Model. Journal of College Student Retention: Research, Theory E Practice, 17(2), 162-185. doi: $10.1177 / 1521025115578230$

Davidson, W. B., Beck, H. P., \& Milligan, M. (2009). The College Persistence Questionnaire: Development and validation of an instrument that predicts student attrition. Journal of College Student Development, 50(4), 373-390. doi: 10.1353/csd.0.0079

Durkheim, E. (2000). O suicídio, estudo de Sociologia. São Paulo: Martins Fontes.

Field, A. P. (2018). Discovering statistics using SPSS (5a. Ed). London, England: SAGE.

Fiuza, P. J., \& Sarriera, J. C.. (2013). Motivos para adesão e permanência discente na educação superior a distância. Psicologia: Ciência e Profissão, 33(4), 884-901. doi: 10.1590/S1414-98932013000400009

Hau, K., \& Marsh, H. W. (2004). The use of item parcels in structural equation modeling: Non-normal data small sample sizes. British Journal of Mathematical Statistical Psychology, 57(2), 327-351. doi: 10.1111/j.2044-8317.2004.tb00142.x

Hu, L. T., \& Bentler, P. M. (1999). Cutoff criteria for fit indexes in covariance structure analysis: Conventional criteria versus new alternatives. Structural Equation Modeling, 6(1), 1-55. doi: 10.1080/10705519909540118

Imperatori, T. K. (2017). A trajetória da assistência estudantil na educação superior brasileira. Serviço Social E Sociedade, (129), 285-303. doi: 10.1590/0101-6628.109

Instituto Nacional de Estudos e Pesquisas Educacionais [INEP] (2016). Censo Escolar da Educação Superior 2016. Brasília. Recuperado de http://portal.inep.gov.br

Jiang, Z. (2017). Social Support and Career Psychological States: An Integrative Model of Person-Environment Fit. Journal of Career Assessment, 25(2), 219-237. doi: 10.1177/1069072715621019

Kline, R. B. (2006). Principles and practice of structural equation modeling (4a ed.). New York: The Guilford Press.

Lacerda, I. P., \& Valentini, F. (2018). Impacto da moradia estudantil no desempenho acadêmico e na permanência na universidade. Psicologia Escolar e Educacional, 22(2), 413-423. doi: 10.1590/2175-35392018022524

Little, T. D., Cunningham, W. A., Shahar, G., \& Widaman, K. F. (2002). To parcel or not to parcel: Exploring the question, weighing the merits. Structural Equation Modeling, 9(2), 151-173. doi:10.1207/S15328007SEM0902_1

Matta, C. M. B., Lebrão, S. M. G., \& Heleno, M. G. V. (2017). Adaptação, rendimento, evasão e vivências acadêmicas no ensino superior: revisão da literatura. Psicologia Escolar e Educacional, 21(3), 583-591. doi:10.1590/2175-353920170213111118

Méndez-Giménez, A., Fernandez-Rio, J., \& Cecchini, J. (2014). Validación de la versión en español del Cuestionario de Metas de Amistad en Educación Física. Universitas Psychologica, 13(1), 227-237. doi: 10.11144/Javeriana.UPSY13-1.vvec

Morosini, M. C., Casartelli, A. O., Silva, A. C. B., Santos, B. S., Schmitt, R. E., \& Gessinger, R. M. (2011). A evasão na educação superior no Brasil: Uma análise da produção de conhecimento nos periódicos Qualis entre 2000-2011. Em Conferencia latinoamericana sobre el Abandono en la educación superior, 1., 2011, Managua. Anais... Madrid: Dpto. de Publicaciones de la E.U.I.T. de Telecomunicación, 2012. p. 65-73.

Nora, A., Barlow, E., Crisp, G. (2005). Student persistence and degree attainment beyond the first year in college. Em A. Seidman, Alan, College Student Retention - Formula for Student Success (pp. 129-154). American Council on Education, Westport: Praeger.

Paula, M. F. C. (2017). Políticas de democratização da educação superior brasileira: Limites e desafios para a próxima década. Avaliação: Revista da Avaliação da Educação Superior (Campinas), 22(2), 301-315. doi: 10.1590/s1414-40772017000200002

Pugh, J., Cramer, J., Slatyer, S., Twigg, D., \& Robinson, M. (2018). Adaptation and pretesting of the College Persistence Questionnaire V3 (Short Form) for measuring intention to persist among Aboriginal Diploma of Nursing students. Nurse Education Today, 61, $162-168$. doi: 10.1016/j.nedt.2017.11.021

Rigdon, E. (1996). CFI versus RMSEA: A comparison of two fit indexes for structural equation modeling. Structural Equation Modeling: A Multidisciplinary Journal, 3(4), 369-379. doi: 10.1080/10705519609540052

Santos Junior, J. S., \& Real, G. C. M. (2017). A evasão na educação superior: O estado da arte das pesquisas no Brasil a partir de 1990. Avaliação: Revista da Avaliação da Educação Superior (Campinas), 22(2), 385-402. doi: 10.1590/s1414-40772017000200007

Spady, W. G. (1970). Dropouts from higher education: An interdisciplinary review and synthesis. Interchange, 1(1), 64-85). doi: 10.1007/ BF02214313 
Tabachnick, B. G., \& Fidell, L. S. (2001). Using multivariate statistics (4a ed.). Boston, MA: Allyn and Bacon.

Tinto, V. (1993). Leaving College: Rethinking the Causes and Cures of Student Attrition (2nd. ed.). University of Chicago Press.

Vautero, J. R, Pozzobon, L. L., \& Zanoello, A. (2009). Democratização na educação Superior - um desafio da política de assistência estudantil. Em La Universidad como objeto de investigación, VI Encuentro Nacional y III Latinoamericano, 2009, Cordoba. La Universidad como objeto de investigación: universidad, conocimiento y sociedad: innovación y compromiso. Cordoba, Argentina.

\section{Sobre os autores}

Jaisso Vautero é psicólogo, PhD em Psicologia Aplicada pela Universidade do Minho, Portugal e psicólogo da Pró-reitoria de Assuntos Estudantis da Universidade Federal de Santa Maria.

Luciane Leorato Pozobon é psicóloga, mestre em Educação pela Universidade Federal de Santa Maria (UFSM) e psicóloga da Pró-reitoria de Assuntos Estudantis da UFSM.

Ana Daniela Silva é psicóloga, PhD em Psicologia Vocacional pela Universidade do Minho, Portugal, Investigadora científica da Escola de Psicologia, Universidade do Minho.

\section{Como citar este artigo}

Vautero et al. (2020). Questionário de Permanência Acadêmica: Adaptação Cultural e Evidências de Validade. Avaliação Psicológica, 19(4), 390-399. http://dx.doi.org/10.15689/ap.2020.1904.17873.05 